\title{
Expression of Vascular Endothelial Growth Factor-C in Oral Squamous Cell Carcinoma: An Immunohistochemical Study
}

\author{
Rakia Sahaf ${ }^{1}$, Aman-ur-Rehman ${ }^{2}$, Sunila Hussain ${ }^{1}$, Ghulam Rasool ${ }^{1}$, Sameer Anjum ${ }^{1}$, Abdul Hanan Nagi ${ }^{1}$ \\ and Nadia Naseem ${ }^{1}$
}

${ }^{1}$ Department of Morbid anatomy and histopathology, University of Health Sciences Lahore, Pakistan

${ }^{2}$ Department of Histopathology, Sheikh Zayed Hospital Lahore, Pakistan

Received: December 18, 2017; Published: January 04, 2018

*Corresponding author: Rakia Sahaf, Morbid anatomy and Histopathology Department, University of Health Sciences, Khayaban-e-Jamia Punjab, Lahore-54600, Pakistan; Email: rakiasahaf@gmail.com

\section{Abstract}

Background: Oral squamous cell carcinoma (OSCC) is globally one of the most frequently reported malignancies characterized by local invasion and distant metastasis. Lymphangiogenesis facilitates invasion and nodal metastasis, and vascular endothelial growth factor $\mathrm{C}$ (VEGF-C) has been implicated as a key mediator of this process. The current study aimed at determining VEGF-C expression in OSCC and its association with Clinicopathological parameters and lymph node metastasis in our population.

Methods: Biopsies from 110 OSCC patients, with detailed documentation of their clinical data were included. After histological examination, cases were evaluated by immunohistochemistry for VEGF-C expression. Reactions were quantified and relation with Clinicopathological parameters explored. The data was analysed using SPSS 21.0.

Results: Mean age of the patients was $59.91 \pm 1.36$ years with the male to female ratio being 1.2:1. OSCC was commonly associated with smoking (30.9\%). Immunohistochemical staining of the histological tissue sections of all cases of well $(\mathrm{n}=42)$, moderate $(\mathrm{n}=38)$ and poorly differentiated OSCC $(n=30)$ revealed extensive VEGF-C immunopositivity compared to normal Keratinocytes. VEGF-C expression was associated significantly with clinical variables $(\mathrm{P}<0.05)$, lymph node metastasis $(\mathrm{P}<0.004)$ and the degree of differentiation of the tumour $(\mathrm{P}<0.001)$.

Conclusion: The findings of the current study underscore the significance of VEGF-C expression in relation to oral carcinogenesis and lymph node metastasis, thereby highlighting its potential for being a promising target for antibody-based therapeutic strategies in Pakistan

Keywords: Vascular endothelial growth factor C (VEGF-C); Lymphangiogenesis; Lymph node metastasis

Abbreviations: OSCC: Oral Squamous Cell Carcinoma; VEGF-C: Vascular Endothelial Growth Factor C; SPSS: Statistical Package for the Social Sciences; WHO: World Health Organization; PBS: Phosphate Buffered Saline; DAB: Di Amino Benzidine

\section{Introduction}

Oral squamous cell carcinoma (OSCC), being the sixth most commonly occurring cancer worldwide [1] is becoming a major global problem and the World Health Organization (WHO) predicts a persistent rise in the incidence of oral cancer. Tobacco, alcohol consumption, betel quid chewing, prevalence of precancerous lesions and traumatic dental history are frequently reported known risk factors associated with OSCC. OSCC is characterized by local invasiveness and high metastatic and recurrence rates. The metastatic dissemination of OSCC preferentially occurs through the lymphatic system. Lymphangiogenesis plays a vital role in tumour growth and systemic dissemination of different carcinomas [2]. Vascular endothelial growth factor C (VEGF-C) is regarded as lymphangiogenic growth factor that has been implicated in inducing the growth of both blood and lymphatic vessels and regulates proliferation, differentiation and migration of lymphatic endothelial cells in many physiological and pathological conditions [3].

It plays a crucial role in the extracellular matrix remodelling and tumour cell invasion by inducing the production of urokinase by cancer [4]. Overexpression of VEGF-C has been detected in various malignancies and is frequently associated with lymphatic invasion, nodal and distant metastasis and consequently poor survival. VEGF-C signalling is involved in the progression of several malignancies that put forward VEGF-C as a potential target for the development of new anticancer therapies to prevent local invasion and metastatic spread of disease. Hence, this study was designed to 
determine the Immunohistochemical expression of VEGF-C in OSCC and to evaluate its association with various Clinicopathological factors and lymph node metastasis of the tumour in Pakistan.

\section{Materials and Methods}

\section{Study Participants}

This descriptive study comprised a total of 110 biopsies of patients who reported with OSCC at different tertiary care hospitals of Lahore, Pakistan, between Jan. 2016- Sep. 2016. Patients from all the age groups and of both genders were enrolled in the study. While those already on radiotherapy, chemotherapy or with a history of comorbidity and/or with recurrence or necrotic tissue specimens were excluded. Written informed consent from the selected patients and an approval from institutional ethical review committee of the University of Health Sciences Lahore, Pakistan (ERC approval vide letter no: UHS/Education/126-15/1776), were obtained prior to the commencement of the study. Demographic and clinical data including age, gender, and history of tobacco or betel quid consumption, tumour location and clinical presentation were collected by reviewing patient records.

\section{Histological Examination}

The specimens were processed for making tissue blocks and sections were cut and stained with haematoxylin and eosin for confirmation of the primary diagnosis. To evaluate malignancy, the histological grading of the specimens was undertaken according to Bryne's classification [5]. The degree of keratinization, nuclear polymorphism, pattern of invasion and inflammatory infiltration were analysed, each with a score varying from 1 to 4 . Total score was calculated by adding the points for each parameter in order to estimate the degree of malignancy of the tumour. Other discrete histological factors, including presence or absence of lymph node metastasis, were also evaluated.

\section{Immunohistochemistry}

About $4 \mu \mathrm{m}$ thick paraffin embedded tissue sections were cut by rotary microtome and collected on Poly-L-lysine coated slides. Sections were deparaffinised in xylene and rehydrated in graded ethanol and were then microwaved in citrate buffer solution ( $\mathrm{pH} \mathrm{6.0)}$ ) for two 5 min cycles in order to retrieve antigens. Standard 'Streptavidin biotin Peroxidase' method was employed for immunostaining. Sections were incubated with rabbit antihuman VEGFC polyclonal antibody (Abcam ab135506, Cambridge, UK; dilution, 1:25) for 1 hour at room temperature. Then they were reacted with biotinylated secondary antibody, followed by streptavidin peroxidase reagent for $30 \mathrm{~min}$ each. All stages were separated by PBS washes, and final visualisation was by DAB chromogen. Sections were counterstained with haematoxylin and finally dehydrated and mounted. Positive (colon cancer) and negative (omission of primary antibody) controls were included in each staining run.

\section{Quantification of VEGF-C Expression}

VEGF-C expression was evaluated using a light microscope by two pathologists, blinded to the information of clinical outcomes. Scoring was done based on the proportion and intensity of staining in tumour cell cytoplasm. The intensity of staining was evaluated as follows: 0 , no staining; 1 , weak; 2 , moderate; and 3 , strong. The proportion of positive tumour cells was scored as 0 (No positive cells); 1 (1-10\% positive cells); 2 (11- 49\% positive cells); 3 (> $50 \%$ positive cells). A combined score for VEGF-C was based on the summation of intensity and the proportion score and was categorized into four groups, where $0=$ No staining; $2=$ weak, $3-4=$ moderate, 5-6= strong [6].

\section{Statistical Analysis}

The data was analysed statistically using SPSS 21.0. Frequencies and percentages were given for qualitative variables and mean \pm S.D were computed for quantitative variables. Pearson Chi square and Fisher Exact test were applied to observe the associations between the variables. AP value of $\leq 0.05$ was considered as statistically significant.

\section{Results}

\section{Clinicopathological Data of Study Population}
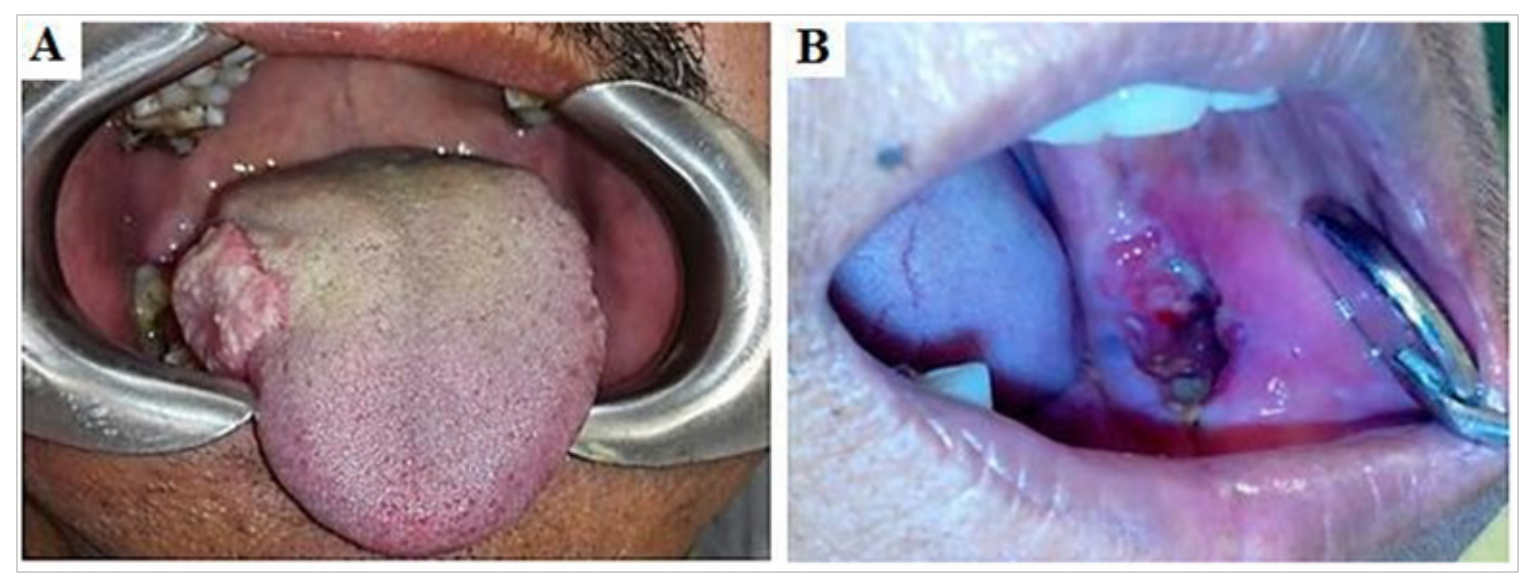

Figure 1: (A) OSCC on the lateral border of tongue presenting as an exophytic mass (B) Ulcerative lesion of OSCC on left buccal mucosa. 
Table 1: Clinicopathological profile of 110 oral squamous cell carcinoma cases

\begin{tabular}{|c|c|c|}
\hline \multirow[b]{2}{*}{ Variables } & \multicolumn{2}{|c|}{ Lymph node Metastasis } \\
\hline & Present & Absent \\
\hline & $\mathrm{n}(\%)$ & $\mathrm{n}(\%)$ \\
\hline \multicolumn{3}{|l|}{ Gender } \\
\hline Male & $21(19.1)$ & $39(35.5)$ \\
\hline Female & $41(37.3)$ & $9(8.2)$ \\
\hline \multicolumn{3}{|l|}{ Age } \\
\hline $25-40$ years & $10(9.1)$ & 0 \\
\hline $41-60$ years & $21(19.1)$ & $29(26.4)$ \\
\hline $61-80$ years & $31(28.2)$ & $19(17.3)$ \\
\hline \multicolumn{3}{|l|}{ Site } \\
\hline Buccal mucosa & $7(6.4)$ & $9(8.2)$ \\
\hline Gingiva & 0 & $10(9.1)$ \\
\hline Lower lip & $5(4.5)$ & $15(13.6)$ \\
\hline Floor of the mouth & $20(18.2)$ & 0 \\
\hline Tongue & $30(27.3)$ & $14(12.7)$ \\
\hline \multicolumn{3}{|l|}{ Etiological factors } \\
\hline Yes & $47(42.7)$ & $31(28.2)$ \\
\hline No & $15(13.6)$ & $17(15.5)$ \\
\hline \multicolumn{3}{|l|}{ Histological grade } \\
\hline Well & $12(10.9)$ & $30(27.3)$ \\
\hline Moderate & $24(21.8)$ & $14(12.7)$ \\
\hline Poor & $26(23.6)$ & $4(3.6)$ \\
\hline
\end{tabular}

A total of 110 OSCC cases were included in accordance with the inclusion and exclusion criteria. Among 110 cases, 54 (49.1\%) OSCC patients presented with lymph nodes metastasis, confirmed by Histopathological analysis. Patient characteristics and histological parameters of the studied cases are summarized in Table 1 The mean age of the study patients was $59.91 \pm 1.36$ years (range: 30-80 years). The male to female ratio was 1.2:1 with most cases reported in 6 th to 8 th decade of life $(p<0.001)$. The age range in males was 30 to 80 years while in females it was 45 to 75 years. Among the 110 biopsies located at variable sites (Figure 1), OSCC predominantly affected tongue (40\%). In females, predominant involvement of tongue was seen $(60 \%)$ whereas buccal mucosa was the commonest site involved in males (26.7\%). Diverse clinical presentations were seen in the current study cases, (Table 2) with most of the patients presenting with an ulcerative lesion $(60.9 \%)$ (Table 1).

Table 2: Expression VEGF-C in oral squamous cell carcinoma (OSCC) and its association with clinicopathologic parameters.

\begin{tabular}{|c|c|c|c|c|c|}
\hline \multirow[b]{2}{*}{ Characteristics } & \multirow[b]{2}{*}{ Total } & \multirow[b]{2}{*}{ Weak } & \multicolumn{2}{|c|}{ VEGF-C expression } & \multirow[b]{2}{*}{$P$ value } \\
\hline & & & Moderate & Strong & \\
\hline & \multirow{2}{*}{ n (\%) } & Positive & Positive & Positive & \\
\hline & & e & n (\%) & $\mathrm{e}$ & \\
\hline & & n (\%) & & n (\%) & \\
\hline \multicolumn{6}{|l|}{ Age (years) } \\
\hline $25-40$ & 10 & 0 & $10(100)$ & 0 & \\
\hline
\end{tabular}

\begin{tabular}{|c|c|c|c|c|c|}
\hline $41-60$ & 50 & 0 & $34(68)$ & $16(32)$ & $<0.001$ \\
\hline $61-80$ & 50 & $10(20)$ & $20(40)$ & $20(40)$ & \\
\hline \multicolumn{6}{|l|}{ Gender } \\
\hline Female & 50 & $10(20)$ & $20(40)$ & $20(40)$ & $<0.001$ \\
\hline Male & 60 & 0 & $44(73)$ & $16(27)$ & \\
\hline \multicolumn{6}{|l|}{ Site } \\
\hline Buccal mucosa & 16 & 0 & $10(62.5)$ & $6(37.5)$ & \\
\hline Gingiva & 10 & 0 & $10(100)$ & 0 & \\
\hline Lower lip & 20 & 0 & $10(50)$ & $10(50)$ & 0.001 \\
\hline $\begin{array}{l}\text { Floor of the } \\
\text { mouth }\end{array}$ & 20 & 0 & $10(50)$ & $10(50)$ & \\
\hline Tongue & 44 & $10(23)$ & $24(54)$ & $10(23)$ & \\
\hline \multicolumn{6}{|l|}{$\begin{array}{c}\text { Clinical } \\
\text { presentation }\end{array}$} \\
\hline Ulceration & 67 & $10(15)$ & $41(61)$ & $16(24)$ & \\
\hline Swelling & 40 & 0 & $20(50)$ & $20(50)$ & 0.004 \\
\hline Exophytic mass & 3 & 0 & $3(100)$ & 0 & \\
\hline \multicolumn{6}{|l|}{$\begin{array}{l}\text { Etiological } \\
\text { factor }\end{array}$} \\
\hline $\begin{array}{l}\text { Betel nut } \\
\text { chewing }\end{array}$ & 23 & $1(4)$ & $9(39)$ & $13(57)$ & \\
\hline Smoking & 34 & $2(6)$ & $15(44)$ & $17(50)$ & $<0.001$ \\
\hline Both & 21 & $1(5)$ & $19(90)$ & $1(5)$ & \\
\hline \multicolumn{6}{|l|}{$\begin{array}{l}\text { Histological } \\
\text { grade }\end{array}$} \\
\hline $\begin{array}{c}\text { Well } \\
\text { differentiated }\end{array}$ & 42 & $2(5)$ & $40(95)$ & 0 & \\
\hline $\begin{array}{l}\text { Moderately } \\
\text { differentiate }\end{array}$ & 38 & $8(21)$ & $10(26)$ & $20(53)$ & $<0.001$ \\
\hline $\begin{array}{c}\text { Poorly } \\
\text { differentiated }\end{array}$ & 30 & 0 & $14(47)$ & $16(53)$ & \\
\hline
\end{tabular}

Regarding the aetiology, $78(70.9 \%)$ patients reported with history of smoking and/or betel quid chewing. Whereas 32(29.1\%) patients had no identifiable risk factors. Smoking was the most common a etiological agent in the present study constituting $34(30.9 \%)$ cases while the simultaneous presence of smoking and betel quid chewing was observed in $19.9 \%$ cases. With regards to histological grade of the tumour, well differentiated OSCC was the most frequent presentation (38.2\%) in the current study cases followed by moderate $(34.5 \%)$ and poorly differentiated carcinoma (27.3\%). In the current study, among the 110 OSCC patients, 62 (56.4\%) cases presented with lymph node metastasis (Figure 2A). The presence of nodal metastasis was higher among the patients with a history of cigarette smoking (37\%). Significant association was instituted between lymph node metastases and histological grades of the tumour $(\mathrm{P}<0.001)$ with most cases of poorly differentiated OSCC $(86.7 \%)$ exhibiting nodal metastasis more frequently then moderate $(63.2 \%)$ and well $(28.6 \%)$ differentiated tumors.

\section{VEGF-C Immunoreactivity}

In the present study, 110 OSCC cases were subjected to immunohistochemistry to assess the proportion and intensity of VEGF-C expression. VEGF-C immunopositivity was detected 
in all OSCC specimens with cytoplasmic staining for the positive tumour cells that ranged from weak to strong positive expression. Conversely, in adjacent normal mucosa, weak to negative immunoreaction was seen (Figure 2B). In OSCC combined score resulting from staining intensities and proportions of positive cells depicted strong positive immunoexpression in 36\% cases
(Figures 3B \& 3C) while moderate expression was noted in $64 \%$ and only $10 \%$ showed weak immunoreactivity to VEGF-C (Figure $3 \mathrm{~A}$ ). VEGF-C expression was detected in the tumour cell nests, with strong positive expression seen in the cytoplasm of invasive tumour cells (Figure 2C).
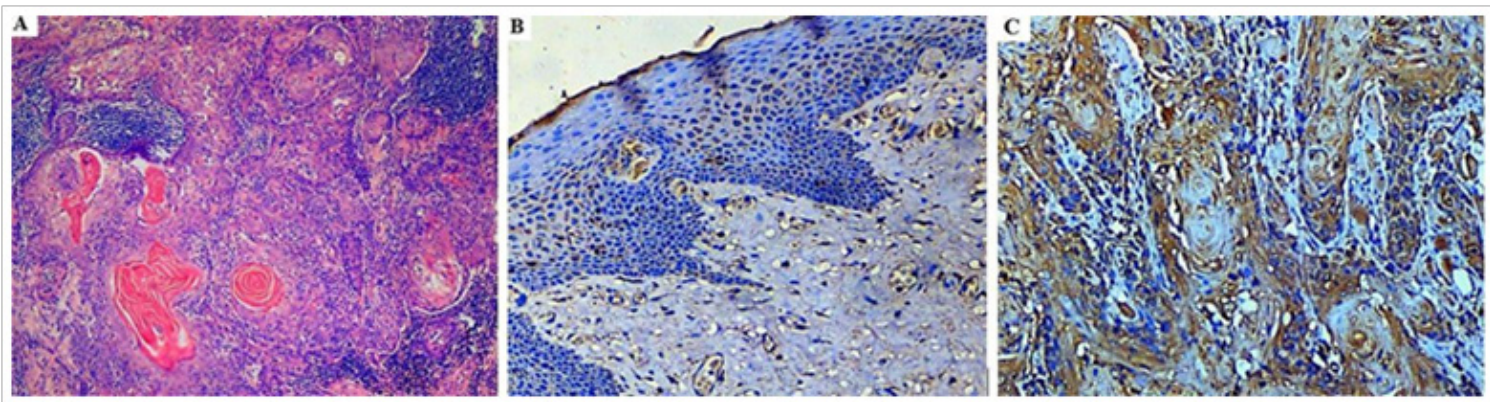

Figure 2: (A) Photomicrograph of lymph node metastasis in a case of moderately differentiated OSCC $(\mathrm{H} \& \mathrm{E}, \times 100)(\mathrm{B})$ Normal oral mucosa showing weak to negative staining for VEGF-C (IHC, x200) (C) OSCC showing strong cytoplasmic expression of VEGF-C in the invasive tumour front (IHC, x200).
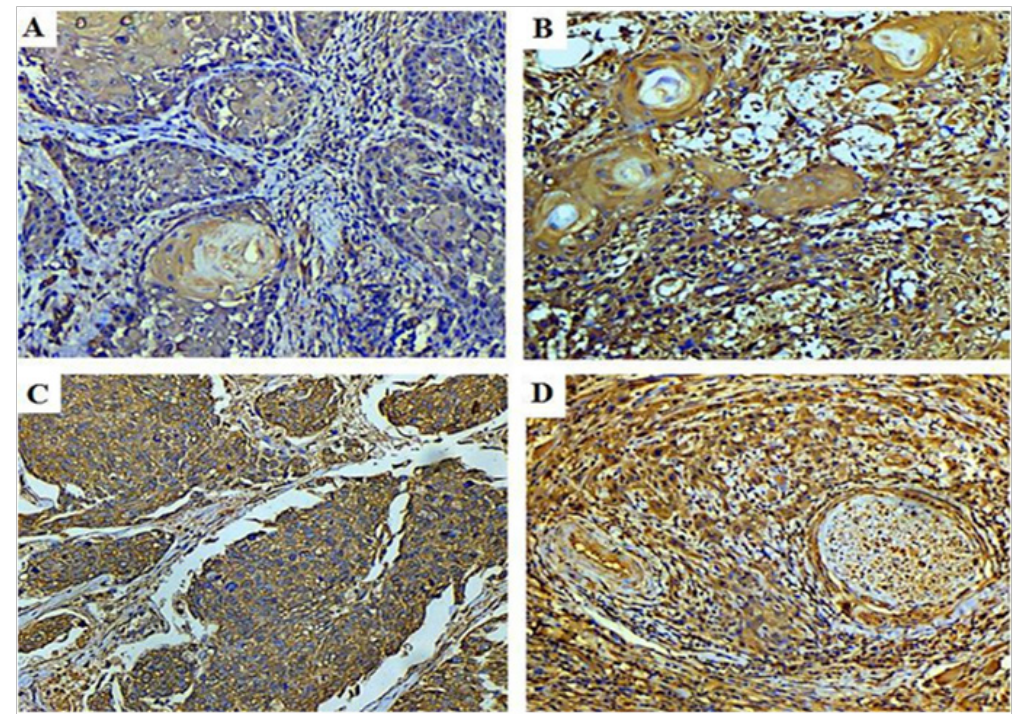

Figure 3: Photomicrograph of (A) well differentiated OSCC showing weak positive cytoplasmic staining (VEGF-C IHC, 200x) (B) Moderate and (C) Poorly differentiated OSCC with strong positive cytoplasmic staining (VEGF-C IHC, 200x) (D): Photomicrograph showing strong VEGF-C staining of tumour cells invading a neurovascular bundle (VEGF-C IHC, 200x).

\section{Association of VEGF-C Expression with Clinicopatholog- ical Parameters}

VEGF-C immunoreactivity with respect to different Clinicopathological parameters of the studied OSCC cases demonstrated a significant relation between VEGF-C expression and age, gender, clinical presentation, etiological factor and location of the tumour $(\mathrm{P}<0.05)$. In different histological grades of OSCC variable VEGF-C immunoreactivity was seen (Figure 2). Moderate positivity (95\%) for VEGF-C was the most frequently observed score in well differentiated OSCC whereas strong positive expression was predominant in moderate $(52.6 \%)$ and poorly differentiated carcinomas $(53.3 \%)(\mathrm{P}<0.001)$. Moreover, the association between the expression of VEGF-C and lymph node metastasis was also found to be statistically significant $(\mathrm{P}=0.005)$ with $61 \%$ cases with lymph node metastasis showing strong positive VEGF-C expression.

\section{Discussion}

Lymphangiogenesis is a crucial requisite for growth and progression of malignant neoplasms. Association of VEGF-C with lymphangiogenesis has provided a likely mechanism for the tumour cells to metastasize through lymphatic's, facilitating invasion and systemic dissemination of tumour. Its high expression in various malignancies has been correlated with tumour progression, nodal and distant metastasis and subsequently poor patient survival [4]. Thus this study was designed to determine the Immunohistochemical expression of VEGF-C in biopsy samples of 
OSCC and its association with Clinicopathological characteristics and lymph node metastasis of the tumour presenting in our part of the world. In future, this study may serve as a benchmark for further assessment of VEGF-C in prognosis and targeted molecular therapy of oral squamous cell carcinogenesis in Pakistan.

In the current study, among the 110 OSCC cases evaluated for different Clinicopathological variables, the age related findings (mean $59.91 \pm 1.36$ years, range $30-80$ years) are consistent with the study conducted by Sato et al. [7] A slight male predilection (Male: Female ratio, 1.2:1) was seen in the present study with the tongue being the most frequently affected site, corroborating data from reviewed literature [8]. In line with the findings of the current study, Syam et al. [9] demonstrated that smoking is the most prevalent etiological agent among the OSCC patients. Consumption of betel quid was more frequent in females while tobacco smoking habit was common in males, which is consistent with other studies [10]. The current study revealed a significant relation between the site of OSCC and lymph node metastasis $(\mathrm{P}<0.001)$ showing that the lesions of tongue were mostly associated with metastasis (48.4\%). These results are consistent with the findings of Youssef et al. [8] and might be explained owing to the rich lymphatic drainage and vascular supply of the tongue.

Regarding the histological grade, amongst the 110 studied OSCC cases, well differentiated carcinoma was the most prevalent presentation (64\%) followed by moderate and poorly differentiated cases. Similar results were seen in local [11] and international studies [12]. According to the immunostaining results of the present study, VEGF-C protein was expressed in all studied cases $(100 \%)$ and was localised to the tumour cytoplasm. From a total of 110 OSCC cases, $\mathrm{n}=36$ demonstrated strong positive expression while $n=64$ and $n=10$ cases revealed moderate and weak VEGF-C immunoreactivity respectively. Similar to these findings, Abdul-Aziz et al. [4] demonstrated high levels of this protein in than $97 \%$ of OSCC cases. The discrepancies in the immunopositivity seen among various studies might be attributed to the different processing techniques and antibodies used, along with the heterogeneity of oral cancer which may be due to the biological/epidemiological disparities between the population and countries. However, the adjacent normal tissue investigated in the present study, depicted weak to negative VEGF-C expression (Figure 2B).

This is in compliance with the findings of Benke et al. [13] who detected markedly upregulated VEGF-C expression in squamous carcinoma cell lines than the normal Keratinocytes. Ming-Xing Ding [14] reported higher expression of VEGF-C mRNA in esophageal carcinoma compared to the normal tissue. This remarkably higher expression compared with the normal tissues support a role for VEGF-C in oral squamous cell carcinogenesis suggesting the use of VEGF-C as a potential biomarker to predict the malignant transformation of oral mucosa. In the current study, significant relationship was seen between VEGF-C Immunohistochemical expression and age, gender, site, clinical presentation of OSCC $(\mathrm{P}<$ 0.05 ). The association of VEGF-C expression and etiological factor of OSCC was also found to be statistically significant exhibiting strong positivity for VEGF-C in the individuals with the history of tobacco smoking (47\%). Akin to these findings, tobacco smoking has also been reported to stimulate VEGF-C expression in cervical carcinogenesis, revealing a significantly higher VEGF-C expression in smokers with cervical intraepithelial neoplasia [15].

The presence of carcinogens in tobacco smoke is a potent stimulator of VEGF-C production. Hypoxia generated by cigarette smoke and HIF-1 $\alpha$ stimulated by nicotine in tobacco plays a critical role in tumour lymphangiogenesis by regulating VEGF-C expression [16]. In the present study, a statistically significant association was seen between VEGF-C expression and degree of differentiation of the tumour $(\mathrm{P}<0.001)$. This finding was also supported by Wang et al. [17] who found the same association in laryngeal squamous cell carcinoma, suggesting that poorly differentiated carcinoma could be more capable of inducing VEGF-C secretion. In the current study, among 56\% OSCC cases presenting with lymph node metastasis, $61 \%$ cases demonstrated strong positive VEGF expression $(\mathrm{P}=0.005)$. In compliance with the findings of Watanabe et al. [18] lymph node metastasis was more common in VEGF-C positive patients, suggesting that being a potent inducer of lymphangiogenesis; VEGF-C promotes lymph node metastasis in cancers. Previously, various studies have reported a correlation between VEGF-C Overexpression and risk of lymphatic metastasis in carcinomas of breast, lung, thyroid, pancreas, prostate and esophagus [19].

Through VEGF-C secretion, primary tumors stimulate dilation of lymphatic vessels and hyperplasia of the sentinel lymph node and increase the number of tumour cells entering the lymphatic's thus contributing to nodal metastasis and tumour progression [20]. In early-stage tongue squamous cell carcinoma, VEGF-C expression was reported as a strong indicator for prediction of lymph node metastases [21] while in advanced stage head and neck squamous cell carcinoma, VEGF-C Overexpression has been correlated to poor patient outcome [22] as in oral cancers the presence of cervical metastasis increases the likelihood of distant metastasis. Therefore, in oral squamous cell carcinoma progression, VEGF-C expression might be regarded as an important factor influencing metastasis to regional lymph node.

Strong VEGF-C expression, associated with positive nodal involvement, may allow the identification of patients who are more susceptible to unfavorable prognosis and permit optimization of therapeutic strategies and offer appropriate treatment to OSCC patients accordingly. Further PCR based studies are also required as a next step to validate these findings. Moreover follow-up studies investigating the impact of VEGF-C Immunohistochemical expression on patients outcome should be conducted in order to determine its relation with disease free/recurrence free survival and prognosis for the patients. This certainly will further improve our understanding of oral SCC tumor genesis and the role played by VEGF-C.

\section{Conclusion}

VEGF-C is extensively expressed by the tumour cells in OSCC patients presenting in our population as compared to the normal tissues. Different etiologic factors of oral cancer may elicit tumour 
lymphangiogenesis via influencing VEGF-C expression. Significant association of VEGF-C protein expression with smoking, lymph node metastasis and degree of histological differentiation highlight the importance of VEGF-C expression in relation to oral carcinogenesis. In this regard VEGF-C might be a promising target for immunotherapeutic approaches, in order to improve morbidity and mortality in OSCC patients in Pakistan.

\section{Acknowledgement}

The authors acknowledge the encouragement extended by the Vice Chancellor of University of Health Sciences, Lahore Pakistan. Also the laboratory staff of Morbid Anatomy \& Histopathology Department of UHS, Lahore for their technical and logistic support

\section{References}

1. Sathiyasekar AC, Chandrasekar P, Pakash A, Kumar KUG, Jaishlal MS (2016) Overview of immunology of oral squamous cell carcinoma. J Pharm Bioallied Sci 8 (Suppl 1): S8-S12.

2. Christiansen A, Detmar M (2011) Lymphangiogenesis and cancer. Genes Cancer 2(12): 1146-1158.

3. Chen JC, Chang YW, Hong CC, Yu YH, Su JL (2013) The role of the VEGF-C/ VEGFRs axis in tumor progression and therapy. Int J Mol Sci 14(1): 88107.

4. Abdul-Aziz MA, Amin AK, El-Rouby DH, Shaker OG (2017) Lymphangiogenesis in oral squamous cell carcinoma: correlation with VEGF-C expression and lymph node metastasis. Int J Dent.

5. Bryne M (1998) is the invasive front of an oral carcinoma the most important area for prognostication? Oral Dis 4(2): 70-77.

6. Abdo MM, Gaballah ET, Fikry HE, Mosbah MM (2014) Expression of VEGF-C in oral precancerous lesions and oral squamous cell carcinoma. Mansoura Journal of Dentistry 1(3):105-108.

7. Sato H, Uzawa N, Takahashi KI, Myo K, Ohyama Y, et al. (2010) Prognostic utility of chromosomal instability detected by fluorescence in situ hybridization in fine-needle aspirates from oral squamous cell carcinomas. BMC Cancer 10(1): 182.

8. Youssef HESM, Aboazma NEE, Megahed EME (2015) Correlation of hypoxia-inducible factor-1 alpha (HIF-1 $\alpha$ ) and vascular endothelial growth factor (VEGF) expressions with clinico-pathological features of oral squamous cell carcinoma (OSCC). Tanta Dent J 12: S1-S14.

9. Sundar SB, Nageswara RR, Faheem MK (2012) Epidemiological and clinico pathological study of oral cancers in a tertiary care hospital. Int J Biol Med Res 3(4): 2376-2380.

10. Akram S, Mirza T, Mirza MA, Qureshi M (2013) Emerging patterns in clinico-pathological spectrum of oral cancers. Pak J Med Sci 29(3): 783787.
11. Khaleel ME, Raza A, Ehsan A, Masood R, Javed M (2015) Clinicopathological spectrum of oral squamous cell carcinoma at a public sector health facility. Biomedica 31(1): 21-26.

12. Krishna A, Singh RK, Singh S, Verma P, Pal US, et al. (2014) Demographic risk factors, affected anatomical sites and Clinicopathological profile for oral squamous cell carcinoma in a north Indian population. Asian Pac J Cancer Prev 15(16): 6755-6760.

13. Benke EM, Ji Y, Patel V, Wang H, Miyazaki H, et al. (2010) VEGF-C contributes to head and neck squamous cell carcinoma growth and motility. Oral Oncol 46(4): e19-24.

14. Ding MX, Lin XQ, Fu XY, Zhang N, Li JC (2006) Expression of vascular endothelial growth factor-C and angiogenesis in esophageal squamous cell carcinoma. World J Gastroenterol 12(28): 4582-4585.

15. Inamine M, Nagai Y, Mitsuhashi A, Nagase S, Yaegashi N, et al. (2012) Cigarette smoke stimulates VEGF-C expression in cervical intraepithelial neoplasia (CIN) 1 and 2 lesions. Int J Clin Oncol 17(5): 498-504.

16. Oliveira-Neto HH, Gleber-Netto FO, De Sousa SF, França CM, Aguiar MC, et al. (2012) a comparative study of microvessel density in squamous cell carcinoma of the oral cavity and lip. Oral Surg Oral Med Oral Pathol Oral Radiol 113(3): 391-398.

17. Wang Z, Chen Y, Li X, Xu Li, Ma W, et al. (2012) Expression of VEGF-C/ VEGFR-3 in human laryngeal squamous cell carcinomas and its significance for lymphatic metastasis. Asian Pac J Cancer Prev 13(1): 2731.

18. Watanabe S, Kato M, Kotani I, Ryoke K, Hayashi K (2013) Lymphatic vessel density and vascular endothelial growth factor expression in squamous cell carcinomas of lip and oral cavity: a Clinicopathological analysis with immunohistochemistry using antibodies to D2-40, VEGF-C and VEGF-D. Yonago Acta Med 56(1): 29-37.

19. Naruse T, Yanamoto S, Yamada SI, Takahashi H, Matsushita Y, et al. (2015) Immunohistochemical study of vascular endothelial growth factor-C/vascular endothelial growth factor receptor-3 expression in oral tongue squamous cell carcinoma: correlation with the induction of lymphangiogenesis. Oncol Lett 10(4): 2027-2034.

20. Liersch R, Hirakawa S, Berdel WE, Mesters RM, Detmar M (2012) Induced lymphatic sinus hyperplasia in sentinel lymph nodes by VEGF-C as the earliest premetastatic indicator. Int J Oncol 41(6): 2073-2078.

21. Matsui T, Shigeta T, Umeda M, Komori T (2015) Vascular endothelial growth factor $\mathrm{C}$ (VEGF-C) expression predicts metastasis in tongue cancer. Oral Surg Oral Med Oral Pathol Oral Radiol 120(4): 436-442.

22. Tanigaki Y, Nagashima Y, Kitamura Y, Matsuda H, Mikami Y, et al. (2004) The expression of vascular endothelial growth factor- $A$ and $-C$, and receptors 1 and 3: correlation with lymph node metastasis and prognosis in tongue squamous cell carcinoma. Int J Mol Med 14(3): 389-395.

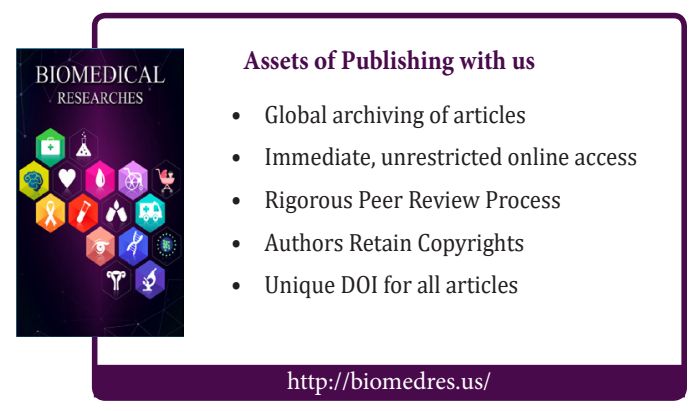

